\title{
La frontera y la televisión
}

\author{
Delia Crovi Druetta, \\ Soledad Robina Bustos y \\ Florence Toussaint Alcaraz
}

Facultad de Ciencias Políticas y Sociales-UNAM

\section{$\dot{\varepsilon}$}

CPOR QUÉ la frontera norte de México ${ }^{1}$ Esta frontera, al compartirse con el país más poderoso del planeta, aparece como una zona compleja pero a la vez claramente definida en el discurso de políticos, periodistas y comunicólogos.

El gobierno mexicano a lo largo de las últimas décadas ha diseñado políticas específicas para esa franja que incluyen planes de desarrollo económico, educativo y cultural. Desde el punto de vista jurídico, el área fronteriza se reduce a una franja de entre 20 y 28 kilómetros dentro del territorio mexicano. Sin embargo, para politólogos y académicos "la realidad fronteriza" se extiende hasta cerca de 200 kilómetros al sur y otros tantos al norte de la línea. La vecindad de México con Estados Unidos le ha dado un sentido especial a su frontera norte. Como en alguna ocasión se ha afirmado: "al rehusarse o afirmarse, ambas naciones se conformaron".

El concepto de frontera lleva en sí la idea de límite. No hay fronteras naturales, hay fronteras históricas que han sido naturalizadas. En este sentido, las fronteras modernas como delimitaciones territoriales cumplen un determinado número de funciones: legales, fiscales, militares, ideológicas, culturales. Formalmente, las fronteras aparecen como un límite a la expresión de la soberanía, estimulan la contradicción entre la legalidad y la ilegalidad.

1 Este artículo fue elaborado a partir de una investigación más amplia, "Televisión y cultura, México y Estados Unidos" que recibió apoyo financiero del Fideicomiso para la Cultura México-Estados Unidos (Fundación Rockefeller, Fundación Cultural Bancomer y Fondo Nacional para la Cultura y las Artes) como parte de su programa cultural de 1994. 
Las fronteras no son sólo la interacción entre el centro y su periferia, son el punto de encuentro entre soberanías nacionales. Aunque el nivel de porosidad entre una frontera y otra puede variar, la proximidad significa un choque de poder entre dos comunidades políticas. Cabe señalar aquí, que sólo en el año 1994 se registraron en puentes internacionales a El Paso 17'239,543 autos y 6'288,628 peatones (El Paso Times, 8 de enero de 1995).

En la frontera no se puede vivir aislado. Ambos lados de la línea se permean con la cultura del "otro". La misma ambigüedad de lo español frente a lo indígena lo sienten los mexicanos frente a la cultura político-económica y la potencia de los Estados Unidos. Esta contradicción no sólo la viven las élites mexicanas. La atracción de lo estadounidense entre las clases populares, se mezcla con el rechazo en esas capas hacia la nación vecina, como es en el caso de la actitud frente al maltrato a los trabajadores indocumentados mexicanos en los Estados Unidos o el rechazo a la Ley 187 que intenta excluir de los servicios sociales, educacionales y médicos a los inmigrantes indocumentados. Esto mismo es válido para las clases media y su encanto por el "american way of life" (Plancarte, 1994). La influencia cultural se refleja a menudo en costumbres cotidianas. Esto significa que para muchos mexicanos, de todos los estratos sociales, el modelo estadounidense constituye un modelo atractivo a la vez que una amenaza permanente. Este fenómeno es aún más complejo en la frontera norte de México.

\section{Y, ¿por qué la televisión?}

En la mayoría de los países del mundo, en menos de cuarenta años, la televisión ha ocupado un lugar predominante en la vida cotidiana de los seres humanos. En Estados Unidos y en el norte de la República Mexicana, como en todas las regiones industrializadas, se ha instalado en casi todos los hogares.

Los medios de comunicación en la era de las nuevas tecnologías ofrecen múltiples posibilidades y no respetan fronteras. Ya nadie puede negar que la prensa, el cine, la radio, el video y hoy, sobre todo la televisión son instrumentos que permean de un modo u otro el modelo de vida de los seres humanos. Así, el desarrollo tecnológico acentúa cada vez más la interdependencia, pero esta interdependencia va también acompañada de muchos desequilibrios y crea desigualdades.

Es difícil aportar datos precisos sobre la penetración que en la actualidad tiene la televisión frente a otros medios. No obstante, hay consenso entre los investiga- 
dores del tema cuando afirman que la radio ha sido poco a poco desplazada por la televisión, principalmente en las comunidades urbanas, aun cuando el número de receptores de radio y de estaciones radiofónicas pueda ser mayor el de los de televisión. Este fenómeno puede explicarse por el desarrollo tecnológico ya que se han multiplicado sus formas de recepción, y con ello su posibilidad de penetración. Hoy en buena parte de los telehogares no sólo se puede encontrar un televisor sino que también, más de una forma de recibir las señales televisivas con contenidos que son verdaderos forjadores de un estilo y modelo de vida que influye de manera significativa en las sociedades modernas.

Es un hecho entonces que los medios de comunicación son vehículos importantes en la interrelación de los países y en particular en las fronteras. El intercambio de valores culturales encuentra un medio privilegiado en la radio y la televisión, intercambio que en el caso de la frontera norte de México es asimétrico como consecuencia de la desigualdad entre un país y otro.

Puede decirse que la mayor parte de lo que cruza las fronteras internacionales es susceptible de imputación de nacionalidad; sin embargo, esta imputación no es tan fácil cuando se trata de elementos culturales (...) en la medida en que este tipo de elementos šn transmitidos más allá de las fronteras por los medios electrónicos de comunicación, las influencias culturales transnacionales se aceleran en favor de las culturas nacionales de países que tienen mayores recursos invertidos en estos medios (Gorostiaga, 1991).

Tijuana-San Diego y Ciudad Juárez-El Paso son ciudades importantes desde el punto de vista de la oferta televisiva ya que su población tiene acceso directo a los mensajes de ambos lados. En este sentido puede afirmarse que para los medios electrónicos la frontera no existe. Esta zona, es también, sin duda una de las zonas donde más señales se reciben en el continente americano.

La investigación sobre cultura y televisión que se desarrolló en la frontera norte constó de cuatro partes. La primera, descrita en este artículo, consiste en una reflexión que aquí esbozamos, sobre la línea divisoria entre México y Estados Unidos, los fenómenos migratorios, los cruces fronterizos, los flujos culturales, la penetración de los medios de comunicación. Después, describimos con amplitud estos medios informativos y de entretenimiento en las dos ciudades más importantes de la frontera norte: periódicos editados, tirajes, índices de lectura; radiodifusoras en AM y FM, frecuencia de sintonía; siguen en esta investigación el teatro, el cine y 
el video, lo cual sirve de marco para contextualizar la enorme influencia que tiene la televisión en esa zona.

En tercer lugar nos centramos en la descripción de la infraestructura televisiva de las ciudades estudiadas, esto es el surgimiento y desarrollo de los canales locales, repetidoras y sistemas de televisión por cable. El análisis de los contenidos de la programación local en Tijuana y Ciudad Juárez del lado mexicano y San Diego y El Paso del lado estadounidense cierra nuestro estudio.

¿La televisión mexicana es igual a la de los Estados Unidos? ¿Cuáles son las diferencias? ¿La televisión de la frontera norte de México y la del Sur de los Estados Unidos están permeadas por la del otro lado? ¿Existe una televisión local fronteriza o sencillamente, una de las zonas que recibe más señales en el mundo acepta un poco más de los mismo? ¿Puede hacerse otro tipo de televisión? Esta son sólo algunas de las preguntas que en este artículo se intentan responder.

En la frontera norte del país confluyen dos fenómenos que convierten a los medios de comunicación en una pieza clave tanto para entender los cambios que la cultura nacional puede sufrir como para percatarse de la evolución de los propios medios, cuya tendencia a largo plazo se extenderá a toda la República. Por el norte entran las innovaciones, se difunde en mayor cantidad con mayor calidad la tecnología de punta y se incia la implantación de modelos pioneros, mismos que vienen de Estados Unidos. Asimismo, gracias al fenómeno de la migración masiva, nuestro vecino del norte está sufriendo una seria influencia de México. Los residentes latinoamericanos en Estados Unidos reclaman de los medios servicios acordes con sus características socio-culturales: idioma español, programas que les recuerden sus raíces, artistas mexicanos, música de su país de origen. Este fenómeno hace de la frontera norte un sitio especialmente interesante para estudiar los hechos emergentes con respecto a la cultura y los medios masivos.

Los datos que se aportan tanto sobre la historia de la televisión como sobre la infraestructura y la programación de las ciudades que nos ocupan son numerosos, casi apabullantes, a lo mejor demasiado minuciosos. Nos limitaremos en este espacio a resaltar lo que nos pareció más interesante.

La información obtenida demostró que de los medios de comunicación-información-entretenimiento, el teatro es el que tiene la menor incidencia entre los habitantes de Tijuana y Ciudad Juárez. De hecho, se registra una butaca de teatro por cuatro de cine. 
El medio que le sigue es la prensa, con índices de lectura bajos, a pesar de ser de los más altos del país. Las cifras indican que el promedio de lectores consuetudinarios de periódicos para el país a principios de los noventa era de 28 por ciento de los habitantes de $\mathbf{1 5}$ años o más, mientras que en la frontera norte el índice era de 42.2 por ciento. También la población alfabeta es mayor en los estados del norte que en el resto de la nación (Lozano, 1991: 16). Aún así, el periódico de la zona que más tiraje registra es ElMexicano que no rebasa los 100,000 ejemplares.

El cine ha adquirido una importancia mayor aunque las salas cinematográficas no satisfacen en su totalidad la demanda de la población fronteriza, lo que ha traído un rápido desarrollo del video. A título de ejemplo podemos mencionar que sólo en Ciudad Juárez se cuenta con cerca de 200 videoclubes en operaciones. Puede destacarse que durante la temporada invernal, probablemente por el clima tan extremoso de la zona, se registran numerosas aperturas de estos establecimientos, asi como un aumento en la compra de videograbadoras.

El número mayor de opciones, en cuanto a las posibilidades de sintonizar canales distintos se encuentra en la radiodifusión. Este es el medio que cuenta con la mayor penetración por número de receptores. Aparentemente, Ciudad Juárez-El Paso es una de las poblaciones más comunicadas de América. En lazona hay 37 estaciones de radio (22 FM y $15 \mathrm{AM}$ ) y 98.9 por ciento de las viviendas tiene por lo menos un aparato de recepción. ${ }^{2}$

Las encuestas apuntan que la ventaja radio-tẻlevisión en cuanto a penetración es relativa en lo que se refiere a las preferencias del auditorio. La pantalla chica aparece entonces como el medio de comunicación más accesible, con una enorme oferta.

Latelevisión es una herramienta que el espectador puede aprovechar de acuerdo con su formación, su gusto y sus necesidades psicoculturales. Sin duda alguna se utiliza fundamentalmente como medio de diversión y para evadirse, ya sea mediante la ficción o los espectáculos y los concursos. Pero también permite a cada uno informarse, adquirir una experiencia concreta del mundo exterior y formarse. Esta fuerza que tiene la televisión es la que nos condujo primero a un exhaustivo recuento de la infraestructura televisiva en la frontera norte de México y después al análisis de los contenidos de la programación de esta televisión.

2 Dátos aportados por Eduardo Barrera, investigador de El Colegio de la Frontera Norte en Ciudad Juárez, febrero 1995. 
Tal como ya se mencionó, la oferta televisiva en Tijuana y Ciudad Juárez es enorme en relación con la media del país. En Tijuana pueden recibirse 14 canales y en Ciudad Juárez 16, si nos referimos exclusivamente a los que pueden captarse con una antena tradicional y "común y corriente". A título de comparación mencionaremos que en la Ciudad de México en el año 1994 se podía tener acceso a 43 canales de televisión. Sin embargo sólo 8 de ellos se recibían por aire, el resto correspondía a lo denominado como "televisión de paga" (Cablevisión, Multivisión, parabólicas).

Una diferencia importante radica en que en la zona metropolitana se paga para recibir las cadenas de Estados Unidos mientras que en Tijuana y Juárez llegan gratuitamente. Lo fronterizo de las ciudades estudiadas hace que se defina su televisión como bilingüe y bicultural.

Si bien Televisa es aún la compañía con mayor presencia en la zona - su señal es difundida a través de los canales locales, repetidoras, cable y parabólicas cadenas como Telemundo en Estados Unidos abren hoy brechas para romper el monopolio que la televisión privada mexicana ha ejercido durante varias décadas. De esta manera las cadenas estadounidenses que transmiten en español compiten ya por la audiencia del público hispanoparlante del otro lado de la forntera. En este sentido se puede reafirmar que la frontera no divide culturalmente ya que las señales de televisión no son objeto de ningún tipo de control a través de ninguna ley o reglamento y circulan con toda libertad.

En cuanto a la programación, se desprende del estudio que los contenidos están en gran parte condicionados por la publicidad. La televisión de la frontera norte se caracteriza también por la ausencia de una televisión estatal, educativa y cultural: todos los canales son comerciales. La influencia de los anunciantes que luchan por ganar mercados de los dos lados de la frontera es determinante para estructurar los mensajes.

Los televidentes reciben así también una publicidad bilingüe y bicultural: la originada en sus ciudades, la proporcionada por las cadenas nacionales y la que viene de Estados Unidos. Los anuncios publicitarios son aquíel reflejo del constante ir y venir de productos culturales y materiales, parte indiscutible de la vida misma de los habitantes de esa región del país.

No está de más señalar que esta publicidad varía según su procedencia y refleja también las desigualdades latentes entre los dos países en otros campos de la vida económica y cultural. Los anuncios locales, desde el punto de vista de la producción, 
son más pobres que los nacionales y más aún que los extranjeros, caracterizados por una mayor calidad en su realización.

Lo primero que resalta del análisis de la programación es la enorme cantidad de horas de transmisión semanales: 3.600 sólo entre Tijuana y Ciudad Juárez. La programación originada en los Estados Unidos llega a casi 63 por ciento mientras que las señales nacionales y locales juntas sólo alcanzan 37 por ciento en el caso de Tijuana y 48 por ciento en el caso de Ciudad Juárez (California's TV,1995 y TV Week, 1995).

A esta desigualdad debe añadirse que de los contenidos de los canales locales de Tijuana, casi 85 por ciento son de origen norteamericano. De tal manera que en realidad no puede hablarse de una televisión fronteriza del norte ya que la producción local es verdaderamente escasa ( 10 por ciento en Tijuana y 20 por ciento en Ciudad Juárez) y el canal con mayor rating en la zona es el Canal 2 de Televisa. Los canales locales se limitan a producir ciertos noticieros y barras noticiosas, programas musicales y deportivos.

Determinar un criterio para analizar los contenidos de la televisión ha sido siempre una tarea compleja que ha llevado a más divergencias que acuerdos. Partiendo de esta premisa, la selección de cinco categorias de análisis convencionales - ficción, información, variedades, deportes, infantiles - ayudó a reconocer la estructura de la televisión. ${ }^{3}$

En cuanto a los contenidos de la televisión en Tijuana y Ciudad Juárezes evidente que la categoría ficción (películas, series, telenovelas) encabeza la lista en cuanto a tiempos de transmisión y se localiza muy por encima de las demás categorías (información, variedades, deportes; infantiles) con 45 por ciento en promedio en las dos ciudades. Lo que confirma nuestra aseveración que la televisión comercial tanto en la frontera como en el resto del país y del mundo es aún medio casi exclusivamente de entretenimiento.

En segundo término, en cuanto a las categorías de programación, se encuentran ligeras variaciones de una ciudad a otra. Mientras que en Ciudad Juárez aparece la categoría información (noticias, opinión) con 23 por ciento, en Tijuana lo hace la de variedades (humor, revistas, musicales, concursos) con 25 por ciento. Siguen

3 Para profundizar en el tema puede consultarse el trabajo de Crovi, Robina y Toussaint (1995): Television y Cultura: El caso de la frontera norte). México: Fideicomiso para la cultura México-Estados Unidos. 
en orden de importancia, en Tijuana información e infantiles (caricaturas, concursos, revistas) y en Ciudad Juárez infantiles y variedades. La programación de las dos ciudades le dedica menor tiempo a deportes (transmisiones, comentarios), ya que esta categoría ocupa la última posición con sólo 5 por ciento del total de horas de la televisión.

Aunque se observan algunas diferencias en los porcentajes de las categorías establecidas en los diversos canales de las ciudades seleccionadas, es importante señalar que la estructura de la programación local de la televisión en la frontera norte es similar en toda la zona y similar también a la del resto del país. Por otra parte, el análisis de la programación de los canales nacionales que se reciben en el norte del país ( 2 de Televisa y 13 de Televisión Azteca en Tijuana) no muestran diferencias sustanciales entre ellos y la programación descrita antes. En los dos casos el mayor número de horas es dedicado a la información, seguido por la categorías ficción, variedades, infantiles y en último lugar deportes. No obstante, durante el fin de semana, dado el conocido interés del consorcio Televisa en este campo, el número de horas dedicadas al deporte aumenta considerablemente.

La televisión comercial se encuentra condicionada en gran medida por la lucha de los mercados y no se dedica a brindar un servicio público. Esto no puede extrañar a nadie ya que la Leyfederal de radio y televisión - que no ha sido modificada desde 1960 , fecha en la que se expidió - favorece a los concesionarios de radioy televisión. El modelo que la televisión comercial desarrolló desde su inicio ha sido funcional para el Estado, eficiente para sus dirigentes.

Televisa además de gozar siempre del apoyo del gobierno federal se concibió siempre, como un negocio que puede producir altos beneficios con inversiones comparativamente pequeñas. Después de cuarenta y cinco años, el concepto parece no haber cambiado, a pesar de la ruptura del monopolio privado y la aparición en el escenario de Televisión Azteca. Hoy en día, la oferta se ha multiplicado, pero esta mayor oferta de mensajes no significa forzosamente una diversidad de contenidos.

El estudio concluye con la descripción de la infraestructura de la televisión en San Diego y El Paso (27 canales transmiten en español o en inglés), así como con el análisis dela programación de las señales transmitidas en esas ciudades. Pareciera que aquí la televisión de Estados Unidos está más centralizada que la de México, ya que los canales locales dejan de serlo para convertirse en repetidoras de o afiliadas a alguna de las cadenas existentes. 
El número de hora de transmisión entre las dos ciudades asciende a 2.136, cantidad bastante menor que la de sus ciudades pares Tijuana y Ciudad Juárez. Los programas con mayor difusión entran también en este caso en ficción y también muy por encima de las siguientes categorías: infommación y variedades, deportes, infantiles y religiosos. Esta última categoría está ausente en el análisis de la programación del lado mexicano por razones obvias: su inexistencia.

A diferencia de la televisión fronteriza mexicana, en la del sur de Estados Unidos puede apreciarse una mayor diversidad de programas entre sus canales. En San Diego y en El Paso la población tiene acceso a un canal estatal educativo-cultural (Public Broadcasting System). Además, según la mercadotecnia del lugar o las alianzas con consorcios de cine se añade o se disminuye el tiempo dedicado a la ficción o la información. Por otro lado, no hay una diversidad real entre la oferta televisiva de una ciudad y otra: menor o mayor acceso a ciertas cadenas, más o menos deportes, más o menos recursos económicos.

En la frontera los mexicanos adoptan pautas culturales de Estados Unidos, pero los norteamericanos adoptan pautas culturales de los mexicanos. La televisión no escapa a esta situación. Sin embargo, las desigualdades saltan a la vista tanto ei el terreno de la infraestructura como en el de los mensajes, especialmente en el de la procedencia de los materiales transmitidos. Los Estados Unidos llenan sus canales en inglés con sus propios materiales, la importación es escasa. No sucede lo mismo con los canales locales mexicanos donde a menudo los materiales importados superan la producción nacional.

El panorama podría parecer desalentador: más opciones pero más de lo mismo. No es así. Es un hecho ya, que la homogeneidad de la televisión estadounidense ha sido resquebrajada no sólo por las señales mexicanas sino también por las transmisiones originadas allá en español (Univisión y Telemundo), hecho que obedece a la percepción del crecimiento de la población hispana en el territorio norteamericano. Por otra parte, no pude olvidarse que la televisión hispana en Estados Unidos se ha extendido en los últimos años fundamentalmente por que los hispanoparlantes a diferencia de otros grupos étnicos minoritarios rechazan la asimilación completa a la sociedad estadounidense. Como grupo no abandonan su cultura, ni su idioma y ésta es una de las razones por las cuales tienden a agruparse en comunidades donde pueden vivir sin perder su identidad cultural.

Pese a la superioridad de los Estados Unidos en materia de televisión, existe en la frontera una resistencia cultural que se manifiesta tanto en la manera de concebir 
al espectador como en las expresiones cada vez más numerosas de rescate de géneros propios. La línea fronteriza es compartida por dos naciones, dos idiomas, dos culturas y un patrón mixto de televisión. El reto para México es no dejarse ganar.

\section{Referencias bibliográficas}

CALIFORNLA'S TV (1995): Vol. II Núm. 12. Tijuana, 14- 20 de enero.

EL PASO TIMEs (1995): El Paso, Texas. 8 de enero.

GoRostiaga Xavier (1991): "América Latina frente a los desafíos globales. Los noventas en una coyuntura estratégica”. Estudios Latinoamericanos Núm. 10. México: UNAM.

LOZANO, José Carlos(1991): Prensa, radiodifusión e identidad cultural en la frontera norte. Tijuana: Colef.

PlanCARTE Roberto (Comp.) (1994): Cultura e identidad nacional. México: Consejo Nacional para la Cultura y las Artes / CFE.

TV WEEK, THE SAN DIEGO UNION TRIBUNE (1995): San Diego, 15-21 de enero. 